\title{
Problems with using large-scale oceanic climate indices to compare climatic sensitivities across populations and species
}

\author{
Martijn van de Pol, Lyanne Brouwer, Lesley C. Brooker, Michael G. Brooker, \\ Diane Colombelli-Négrel, Michelle L. Hall, Naomi E. Langmore, Anne Peters, \\ Stephen Pruett-Jones, Eleanor M. Russell, Michael S. Webster and Andrew Cockburn
}

M. van de Pol (martijn.vandepol@anu.edu.au), L. Brouwer, N. E. Langmore and A. Cockburn, Evolution, Ecology and Genetics, Research School of Biology, Australian National Univ., Canberra, ACT 0200, Australia. - L. C. Brooker and M. G. Brooker, 27 Marion Way, Gooseberry Hill, WA 6076, Australia. - D. Colombelli-Négrel, School of Biological Sciences, Flinders Univ., GPO Box 2100, Adelaide 5001, Australia. - M. L. Hall, Dept of Zoology, Univ. of Melbourne, Melbourne, VIC 3010, Australia, and Max Planck Inst. for Ornithology, Vogelwarte Radolfzell, DE-78315 Germany. - A. Peters, Max Planck Inst. for Ornithology, Vogelwarte Radolfzell, DE-78315 Germany, and School of Biological Sciences, Monash Univ., VIC 3168, Australia. - S. Pruett-Jones, Dept of Ecology and Evolution, Univ. of Chicago, 1101 East 57th St., Chicago, IL 60637, USA. - E. M. Russell, 53 Swan Street, Guildford, WA 6055, Australia. - M. S. Webster, Dept of Neurobiology and Behavior and Cornell Lab of Ornithology, Cornell Univ., Ithaca, NY 14853, USA.

\begin{abstract}
To understand which populations and species are most sensitive to climate change, studies correlate time series of climate variables with those of traits important for population dynamics, and subsequently compare which aspects of a species' ecology or life-history best explain variation in climate sensitivity. Often large-scale oceanic climate indices (LOCIs) are used as a proxy for local climatic drivers, with many studies reporting geographic gradients in climate sensitivity to LOCIs (e.g. suggesting that species living further from the equator are relatively climate sensitive). However, the relationship between LOCIs and local weather variables also varies geographically, raising the possibility that apparent intra- and inter-specific differences in climate sensitivity to LOCIs could also reflect geographic variation in how well LOCIs function as a proxy for local climatic drivers. This hypothesis is rarely tested due to lack of knowledge about the specific local climatic drivers. Here we show, using reproductive and climate data from 16 long-term population studies of 7 Australian fairy-wren species (Malurus genus), that the use of LOCIs can result in 1) strong overestimation of the amount of inter-specific variation in climate sensitivity and 2) spurious patterns, particularly geographic gradients. Consequently a paradox emerges: LOCIs often explain much of the temporal variation in traits important for population dynamics, but the common usage of LOCIs may prevent meaningful intra- and inter-specific comparisons of climate sensitivities over large spatial scales. Our results thus may offer an alternative interpretation of the widely reported geographic gradients in sensitivity to LOCIs. Future progress will likely require better knowledge about the identity and temporal features of local environmental drivers of population dynamics.
\end{abstract}

Responses to climate change and variability have been widely documented (Forchhammer et al. 1998, Parmesan and Yohe 2003) and predictions of their consequences are being made both on the scale of local populations (Jenouvrier et al. 2009, van de Pol et al. 2010, 2011) and entire range distributions (Thomas et al. 2004, Keith et al. 2008). A major aim for climate change ecologists is to understand which populations and species are most sensitive to climate variability and change and why this is so. To this end, studies cross-correlate time series of a climatic variable to time series of traits important for population dynamics, such as phenological (Sanz 2002, 2003, Rainio et al. 2006, Macmynowski et al. 2007, Phillimore et al. 2010), demographic (Grosbois et al. 2006, Sandvik and Erikstad 2008, Sandvik et al. 2008) and life-history traits (Mysterud et al. 2000, Martinez-Jauregui et al. 2009, Gardner et al. 2011), range shifts and contractions (Amano and Yamaura 2007, Chen et al. 2011), as well as population sizes and growth rates (Sæther et al. 2003, Anders and Post 2006, Lima et al. 2008, Sandvik et al. 2012). The increasing availability of comparable long-term datasets on different populations and species means that ample opportunities exist to perform formal comparative analysis on which aspects of the phylogeny, distribution and biology of a species best explain variation in climate sensitivity. Such associations can be used to derive much needed generalisations in climate change ecology (Sandvik and Erikstad 2008).

Many studies that compare the climate sensitivity of traits across populations or species have used large-scale oceanic indices (LOCIs) as the climatic variable of choice, most notably the El Niño/La Niña-Southern Oscillation (ENSO) index and the North Atlantic Oscillation (NAO) 
index (Post et al. 1997). Our ecological knowledge about the timing, spatial scale and characteristics of the local weather processes causally affecting trait expression is often poor (Hallett et al. 2004, van de Pol and Cockburn 2011), and we have to rely on LOCIs as a proxy. Remarkably, in practice LOCIs often explain more of the variation in key traits than single local weather variables (Post and Stenseth 1999, Stenseth and Mysterud 2005). This could arise because LOCIs function as an appropriate 'weather package': they integrate the temporal and spatial features of several weather components (i.e. temperature, precipitation, wind) into a single variable (Hallett et al. 2004, Stenseth and Mysterud 2005).

Assessing climate sensitivity across populations and species typically involves comparisons over large spatial scales. However, due to the inherent large scale nature of LOCIs, the relationship between LOCIs and local weather inevitably shows strong geographic variation (see Hurell and Trenberth 2010 for an overview), sometimes even on a small spatial scale (Mysterud et al. 2000). This raises the possibility that apparent differences in climate sensitivity to LOCIs across populations and species do not necessarily reflect biologically meaningful variation, but instead reflect geographic variation in how well LOCIs function as a proxy for the local climatic drivers (Fig. 1). This hypothesis is of particular interest given that by far the most frequently invoked explanation for intra- and inter-specific variation in climate sensitivity is the existence of geographic gradients, mostly latitudinal (Sæther et al. 2003, Sanz 2003, Anders and Post 2006, Lima et al. 2008, Sandvik et al. 2008, 2011), but sometimes longitudinal (Sanz 2002, 2003) and altitudinal (Mysterud et al. 2000). By contrast, there is little empirical support for effects of phylogeny, life-history or other aspects of the ecology of a species on its climate sensitivity (but see Sandvik et al. 2012).

The hypothesis that geographic variation in the correlation between LOCIs and local weather may explain intraand inter-specific differences in climate sensitivity to LOCI is occasionally acknowledged in the literature (Sandvik et al. 2008). However, explicit tests are rare because detailed knowledge about the identity and critical period of the local weather variables that drive trait variability is required for a large number of populations and/or species. Studies that have attempted to do so found mixed results: Anders and Post (2006) found evidence that differences across populations in climate sensitivity of bird densities to NAO and ENSO were due to spatial heterogeneity in how these LOCIs predicted local air temperatures. Similarly, Mysterud et al. (2000) showed in great detail how the differential effect of $\mathrm{NAO}$ on the performance of deer along an altitudinal gradient could be understood from the fact that NAO is correlated with less snow at low altitude, and more snow at high altitude. By contrast, Sandvik et al. (2008) suggested that differences across seabirds in climate sensitivity of demographic rates to NAO were not solely due to geographic variation in how well NAO predicted local sea-surface temperatures.
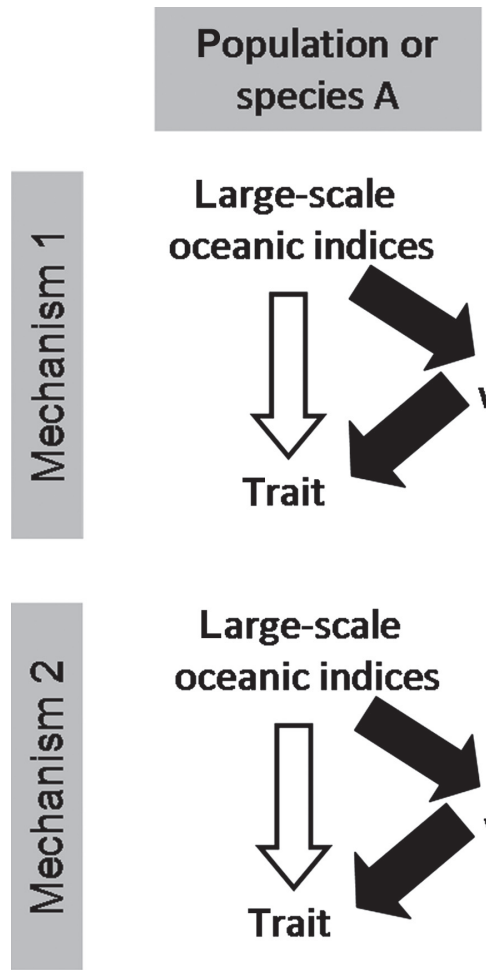
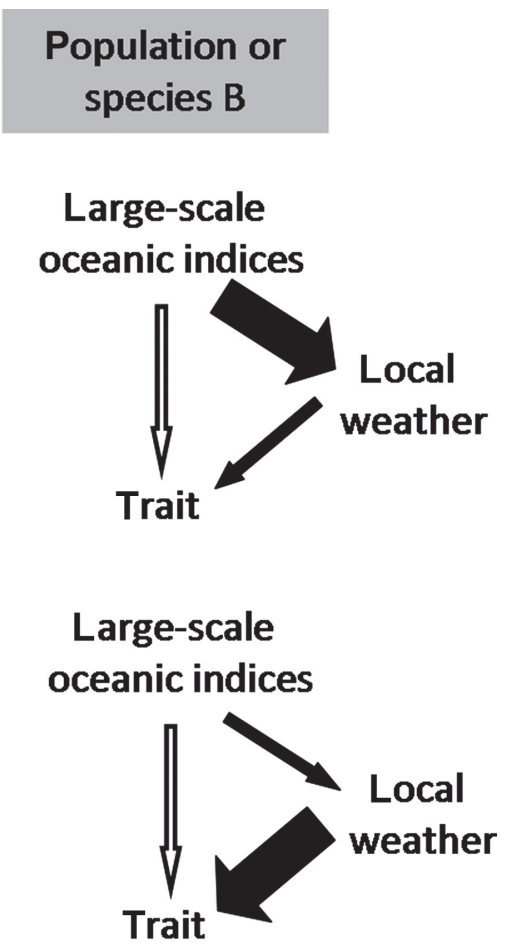

Figure 1. When comparing the climate sensitivities between populations or species that differ in geographic location (A vs B), variation in observed sensitivities to large-scale oceanic indices (white arrows of different thickness) may result from two different mechanisms (black arrows of different thickness), 1) genuine variation in climate sensitivity or 2) from geographic variation in the strength of the association between large-scale oceanic indices and the local weather variables that are actually responsible for changes in traits. Most studies interpret observed differences between populations/species in climate sensitivity to LOCIs by assuming that the first (biological) mechanism is at work, while the alternative (non-biological) explanation is difficult to refute without detailed knowledge about the local weather variables that drive trait change. 
The aim of this study is to test whether variation among populations and species in climate sensitivity to a LOCI can be explained by geographic variation in how well this LOCI predicts the local weather conditions that drive trait variability. Furthermore, we will for the first time explicitly investigate two important corollaries. First, does the usage of LOCIs cause overestimation of the amount of variation among populations or species in climate sensitivities? This can be expected, since at least some of the apparent variation in climate sensitivity to LOCIs could be due to the variable relationship between LOCIs and local weather variables. Second, do geographic gradients in climate sensitivity to LOCIs reflect a similar geographic gradient in climate sensitivity to local climatic drivers? We will address these aims using long-term demographic data from 16 populations of 7 Australian fairy-wren species. Reproductive success of these insectivorous passerine birds of the Malurus genus strongly depends on rainfall before and during the breeding season (Rowley and Russell 1993, Russell and Rowley 2000, Brooker and Brooker 2002, Webster et al. 2010, van de Pol et al. 2012) and we will therefore compare its sensitivity to the local rainfall as well as to two regionally influential large-scale oceanic climate systems: the Pacific Ocean ENSO and the Indian Ocean Dipolar Mode index (DMI; Saji et al. 1999).

\section{Methods}

\section{Reproductive data on fairy-wrens}

We collated published and unpublished data from 16 populations of 7 cooperatively breeding species of Australian fairy-wrens (see Supplementary material Appendix 1 for an overview). Our dataset included six populations of $M$. cyaneus (Rowley 1964, Nias and Ford 1992, Cockburn et al. 2008, Colombelli-Négrel and Kleindorfer 2008, Langmore et al. 2009), two populations each of $M$. coronatus (van Doorn 2007, Kingma et al. 2009), M. melanocephalus (Webster et al. 2010), M. pulcherrimus (Brooker and Brooker 2002, Rowley and Russell 2002) and M. splendens (Rowley et al. 1991, Pruett-Jones et al. 2010), and one population each of $M$. elegans (Brouwer et al. 2011) and M. leucopterus (Rowley and Russell 1995). Studies were selected on the condition that they had collected data on annual reproductive success (ARS; the average number of fledglings per territory per year) for a period of at least three years (range 3-38; mean $9 \mathrm{yr}$ ). The earliest data are from 1956, while most data are from the period 1980-2011.

\section{Climatic data}

Data on monthly rainfall were available from the Australian Bureau of Meteorology (<www.bom.gov.au/climate/ $>$ ). For most of the 16 study sites we used data from the nearest weather station, however if no weather station was within a $50 \mathrm{~km}$ radius we used the average rainfall of the two to three nearest weather stations, where values were weighted proportional to the proximity to the study site.
Rainfall in Australia is influenced by the large-scale climate systems of both the Indian and Pacific Ocean (Risbey et al. 2009, Cai et al. 2011), and we therefore considered both the DMI (Saji et al. 1999) and the ENSO index (Hurell and Trenberth 2010) as LOCIs that may serve as a proxy for local rainfall patterns (data sources: Climate Prediction Center 2012, OOPC 2012). Historically El Niño/La Niña events were thought to be the key driver of Australian rainfall patterns, but awareness is now growing that the Indian Ocean Dipole is at least as important. Both climate systems are in fact related to each other over large distances (i.e. teleconnected), and the DMI and ENSO index are strongly intercorrelated, especially during spring (Cai et al. 2011; i.e. the breeding season of most populations). The ENSO index is thought to primarily predict rainfall variability in near-tropical eastern Australia, while extra-tropical rainfall patterns during $\mathrm{El}$ Niño events are thought to be more strongly related to the DMI (Cai et al. 2011).

Preliminary analysis showed that DMI explained substantial amounts of the within-population temporal variation in ARS (maximal reduction of deviance by 17 units, depending on months over which DMI was averaged), while ENSO explained little additional variation (maximal reduction of model deviance by 4 units; Supplementary material Appendix 2). We therefore henceforth focus on the role of DMI as a proxy for local rainfall, but emphasize that due to quite high intercollinearity $(r<-0.5)$ the effects of DMI and ENSO are hard to disentangle statistically and that therefore the interpretation of their climate sensitivity is interrelated.

A low DMI is typically associated with high rainfall. The correlation between DMI and rainfall in winter and spring is known to vary geographically (Risbey et al. 2009), with a strong latitudinal component due to strongly negative correlations in the south and west of Australia and close-to-zero correlation in most of (tropical) north Australia (Fig. 2). The 16 sites included in our dataset were situated along key parts of this geographic gradient (Fig. 2) and consequently the 16 sites varied widely in their correlation of DMI and local rainfall (range -0.06 to -0.47 ).

\section{Statistical analysis of climate sensitivity}

We define the climate sensitivity of a population as the correlation between ARS and a climatic variable, either DMI or local rainfall amounts. A highly positive or negative correlation is interpreted as strong climate sensitivity and a close to zero correlation as weak climate sensitivity. To determine the overall amount of variation explained by the climatic variables, we used a general linear mixed model (LMM; ARS was approximately Gaussian distributed) in which a population's identity was entered as a random intercept term and in which we also allowed the relationship between ARS and climatic variables to vary among populations using a random slope term. The climatic variables were withinpopulation centred to ensure that the relationships would reflect within-population associations (van de Pol and Wright 2009). To account for uncertainty arising from variation in sample size across years within studies we 


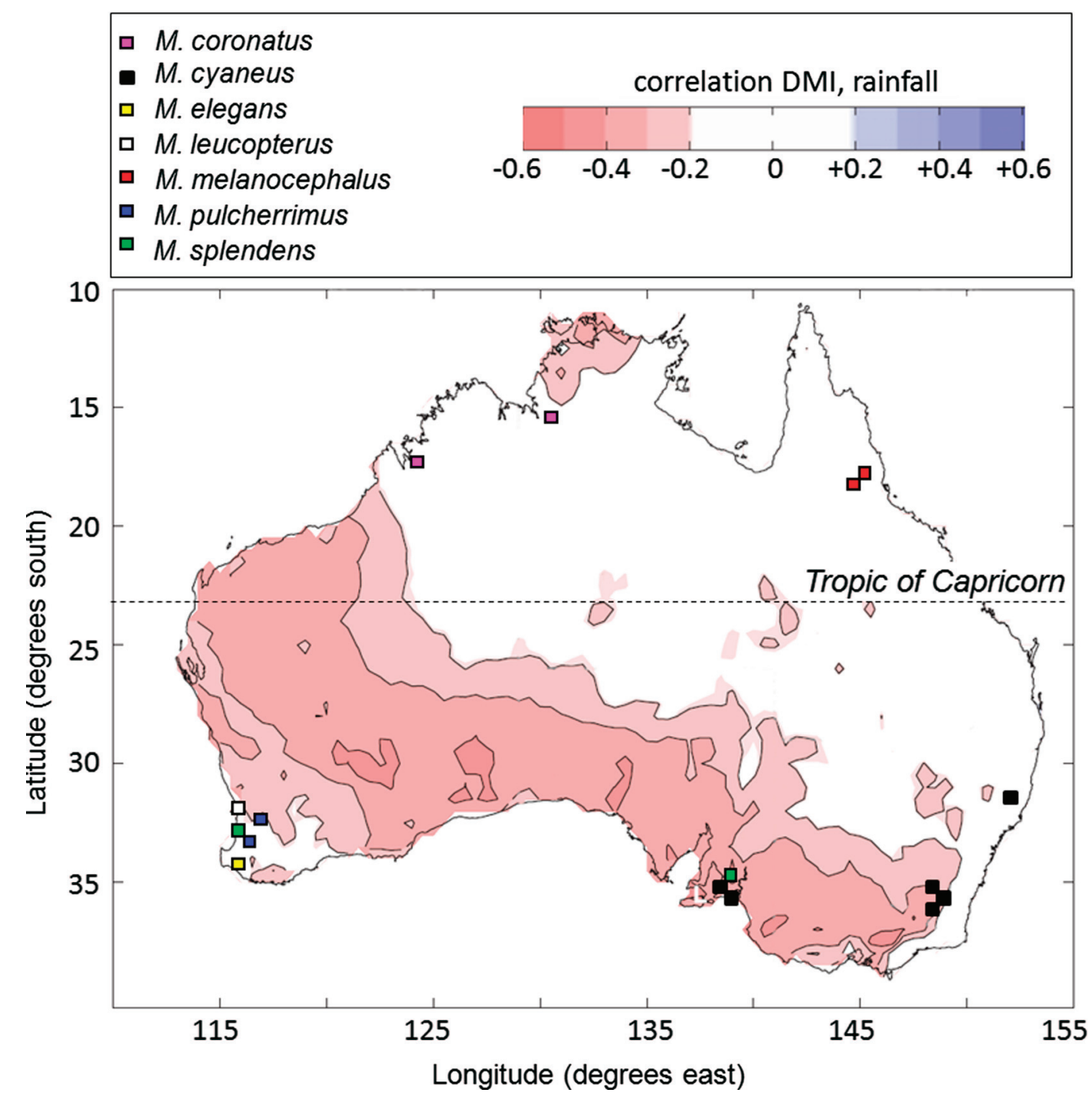

Figure 2. Locations of fairy-wren (Malurus genus) populations included in the comparative analysis. The correlation between the Indian Ocean dipolar mode index and mean winter-spring rainfall across the Australian continent is shown by the intensity of shading (Risbey et al. 2009).

included the square root of the number of territories for which reproductive success was sampled in a given year as a weight in the LMM. Furthermore, a species' identity was entered as a random intercept term to account for potential phylogenetic differences.

Although previous studies on individual populations suggested that high rainfall during winter, spring and/or summer amplified reproductive success (Rowley and Russell 1993, Russell and Rowley 2000, Brooker and Brooker 2002, Webster et al. 2010, van de Pol et al. 2012), we performed a sliding window approach (van de Pol and Cockburn 2011) to determine a more exact period of the year over which rainfall and DMI explained most of the variation in ARS. To avoid overfitting we assumed the same time window for all populations; this assumption is hard to verify, but similar assumptions are always - often implicitly - made in comparative analysis and we will return to this point in the Discussion.
To determine what geographic factors (latitude, longitude) could explain variation among populations in climate sensitivity, we performed weighted regression analysis in which the climate sensitivity of a population was the unit of analysis. The contribution of each study to the overall model fit was weighted proportional to the square root of the duration of the study to account for inherent inaccuracy resulting from short study periods (sensu Sandvik et al. 2008). Analyses were performed using R 2.14 (R Development Core Team) using two-tailed test statistics.

\section{Results}

A sliding window approach showed that rainfall and DMI over the period April-December best explained withinpopulation variation in ARS (Supplementary material Appendix 2), and climate variables were averaged over 
this critical period in all subsequent analyses. Although DMI explained a substantial amount of the temporal withinpopulation variation in ARS $\left(\mathrm{R}^{2}=0.13\right.$, joint test of fixed and random component of DMI in LMM: $\chi_{\mathrm{DF}=2}^{2}=12.9$, $\mathrm{p}=0.002)$, local rainfall explained twice as much of the variance $\left(\mathrm{R}^{2}=0.25\right.$, joint test of random and fixed component of rainfall in LMM: $\chi_{\mathrm{DF}=2}^{2}=14.5, \mathrm{p}<0.001$ ), suggesting that DMI is a reasonable proxy and that local rainfall is likely a key local climatic driver.

Higher than average rainfall was strongly associated $(r=0.24-0.90)$ with above average ARS in all but the two shortest population studies (i.e. $\leq 4 \mathrm{yr}$ of data). Lower than average DMI was strongly associated $(-r=0.22-0.91)$ with above average ARS in 11 populations, but in five populations the climate sensitivity to DMI was weak or in the opposite direction (Supplementary material Appendix 1).

The strength of the correlation between DMI and rainfall explains much of the variation among populations in climate sensitivity to $\mathrm{DMI}$ (weighted regression $\mathrm{F}_{\mathrm{DF}=1,14}=$ 9.3, $\mathrm{p}=0.009, \mathrm{R}^{2}=0.36$ ). The trendline suggests that populations in which DMI was most negatively correlated with rainfall (low DMI is associated with high rainfall; Fig. 2) were also the populations with the strongest climate sensitivity to DMI, while populations in which DMI did not predict rainfall did not respond to DMI in the expected direction (Fig. 3a).

Populations of fairy-wrens varied $60 \%$ more in their climate sensitivity to DMI than in their climate sensitivity to rainfall (weighted variances respectively 0.16 and 0.10 ). A latitudinal gradient was present for the climate sensitivity to DMI (weighted regression $\mathrm{F}_{\mathrm{DF}=1,14}=7.2, \mathrm{p}=0.018$, $\mathrm{R}^{2}=0.29$ ), with tropical populations typically being less climate sensitive to DMI than populations from temperate regions (Fig. 3b). By contrast, there was no evidence of a latitudinal gradient in climate sensitivity to rainfall (Fig. 3c; weighted regression $\mathrm{F}_{\mathrm{DF}=1,14}=0.1, \mathrm{p}=0.85, \mathrm{R}^{2}=$ $0.00)$. These results were qualitatively similar when we added species identity as a random intercept in the regression model to account for the fact that climate sensitivities - especially to DMI - of the same species seemed intercorrelated.

\section{Discussion}

We showed that Australian fairy-wren populations vary strongly in their climate sensitivity to the large-scale oceanic index DMI. We also showed that local rainfall explained more of the between-year variation in reproductive success than did DMI; local rainfall is thus likely to be a key local climatic driver of reproduction and thereby population dynamics in fairy-wrens. Furthermore, differences among Australian fairy-wren populations in climate sensitivity to DMI strongly depended on how well DMI predicted local rainfall. Together these results imply that much of the observed variation in climate sensitivity to the LOCI DMI is not due to biologically relevant differences between populations or species, but rather an artefact of the use of LOCIs and the fact that DMI varies geographically in how well it functions as a proxy for rainfall. A similar outcome was reported for the climate sensitivity of North-American yellow-billed cuckoo Coccyzus americanus
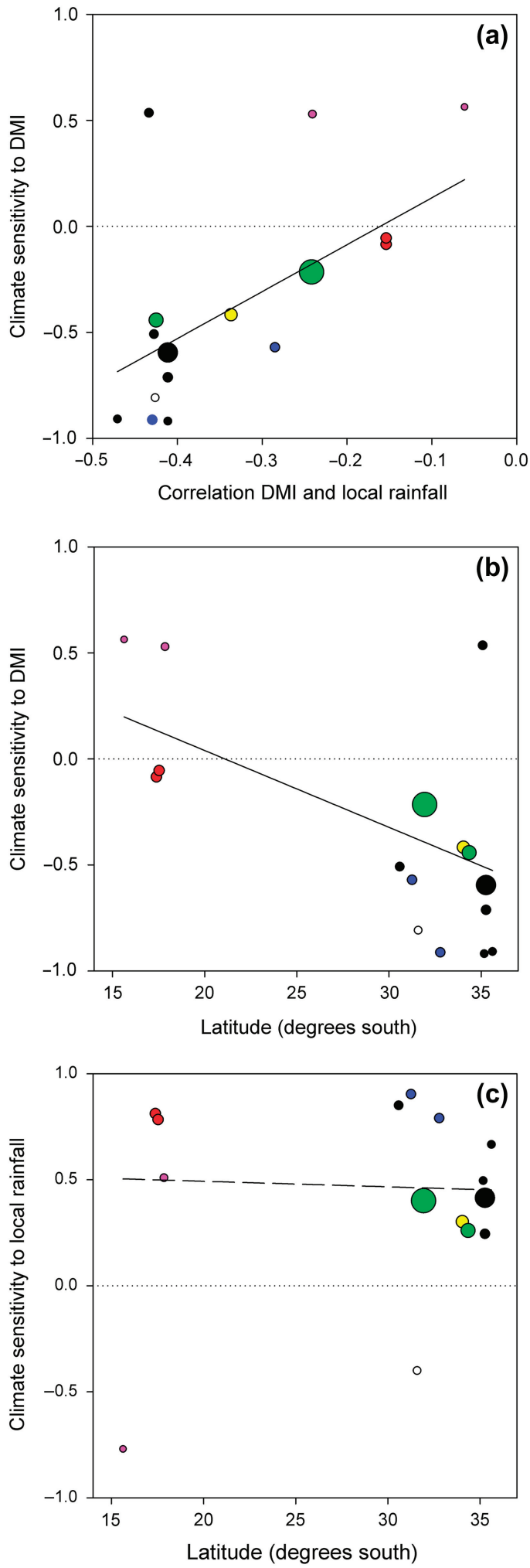

Figure 3. Relationships between (a) climate sensitivity of annual reproductive success to DMI and the strength of the correlation between DMI and local annual rainfall, (b) climate sensitivity of annual reproductive success to DMI and latitude, (c) climate sensitivity to local rainfall and latitude. Each circle represents one population, with the size of the circle being proportional to the square root of the length of the study (ranging 3-38 yr) and colours differing among species (see legend of Fig. 2); some points were slightly jittered to reduce visual overlap of symbols. 
numbers to NAO and ENSO (Anders and Post 2006), and to some extent also for the climate sensitivity of the demographic rates of Atlantic seabirds' to NAO (Sandvik et al. 2008).

In our study we have assumed that the amount of local rainfall averaged over the period April-December is a key causal driver of between-year variation in reproductive output. This assumption was supported by the result that rainfall over this period explained $25 \%$ of the betweenyear variation in ARS within populations. The explanatory power of rainfall would possibly have been higher if we had better knowledge about differences among populations in the critical period over which the rainfall affects reproduction. Furthermore, other local weather variables, such as air temperature may further affect reproductive success. Thus our focus on local rainfall from April to December is potentially only part of the story (this will be explored elsewhere). However, our aim here was not primarily to determine the true climate sensitivity - which is arguably very hard - but instead to illustrate that climate sensitivities to LOCIs are not reflected by climate sensitivities to a local weather variable which we know explains at least a substantial amount of variation in the trait of interest.

Our study is the first to present direct evidence for two important corollaries of the common use of LOCIs in comparative analysis of climate sensitivities over large scales. We showed that the use of LOCIs to compare climate sensitivity among populations can result in 1) systematic bias in the amount of variability and 2) spurious associations among populations in climate sensitivity. High intra- and inter-specific variation in climate sensitivity may lead us to conclude that it is hard to predict and generalize how different populations and species will respond to climate change and variability. Spurious relationships among populations and species in climate sensitivities, such as latitudinal gradients may cause us to believe erroneously that for example tropical birds are less climate sensitive than birds from temperate regions (Fig. 2, 3). Potentially, it could also cause spurious associations with life-history, ecological and phylogenetic characteristics, since such characteristics also often show geographical patterns such as latitudinal gradients (Lack 1947, Blanckenhorn and Demont 2004). Finally, identifying the correct underlying mechanism causing geographic variation in climate sensitivity will be important for understanding the spatial synchrony - or lack thereof - in temporal aspects of demography and population dynamics across a species' distribution, which in turn determines meta-population dynamics.

To reduce the impact of geographic variation in LOCIlocal weather correlations on estimates of climate sensitivity to a LOCI, Sandvik et al. $(2008,2012)$ suggested ignoring the sign of climate sensitivity by focussing for example on the coefficient of determination $\left(\mathrm{R}^{2}\right)$ instead of the correlation coefficient as a measure of climate sensitivity. This approach may eliminate some of the apparent variation in climate sensitivity to LOCIs when the sign of the relationship between LOCIs and local weather variables varies geographically (as it does for NAO and winter temperature in Europe; Hurell and Trenberth 2010). However, their approach does not filter out spurious variation in climate sensitivity that arises because the relationship between LOCIs and local weather variables can be present in some areas, but absent (i.e. zero) in others (as in Fig. 3a). For example, the correlation (and $\mathrm{R}^{2}$ ) of two populations of $M$. melanocephalus to DMI was estimated to be almost zero (red dots Fig. 3b), while its climate sensitivity to local rainfall was strongly positive (red dots in Fig. 3c, $\mathrm{R}^{2}>0.6$ ).

In conclusion, a paradox emerges: the literature clearly shows that LOCIs often explain much of the variation in traits important for population dynamics, but our study suggests that precisely this common usage of LOCIs may prevent meaningful comparisons of climate sensitivities across species over large areas. We thus need better ecological knowledge about the (multivariate) identity and spatiotemporal features of local environmental drivers of population dynamics, which is likely to be a daunting task (Hallett et al. 2004, Stenseth and Mysterud 2005, van de Pol and Cockburn 2011). Finally, our results may provide an alternative interpretation of the widely reported geographic gradients in sensitivity to LOCIs in the literature (see references in Introduction).

Acknowledgements - Author contribution: MvdP developed the idea, performed the analysis and wrote the paper, LB discussed the analysis and results, all authors contributed to data collection/ collation and commented on the manuscript. We thank all other people that contributed to data collection in each of the studies over the years and Misty Barron and Wendy Dimond for database services. MvdP was supported by an Australian Postdoctoral Fellowship (DP1092565), NEL by an Australian Research Fellowship of the Australian Research Council, and AP by a Minerva Fellowship of the Max Planck Society and an ARC Future Fellowship (FT110100505).

\section{References}

Amano, T. and Yamaura, Y. 2007. Ecological and life-history traits related to range contractions among breeding birds in Japan. - Biol. Conserv. 137: 271-282.

Anders, A. D. and Post, E. 2006. Distribution-wide effects of climate on population densities of a declining migratory landbird. - J. Anim. Ecol. 75: 221-227.

Blanckenhorn, W. U. and Demont, M. 2004. Bergmann and converse Bergmann latitudinal clines in Arthropods: two ends of a continuum? - Int. Comp. Biol. 44: 413-424.

Brooker, L. C. and Brooker, M. G. 2002. Dispersal and population dynamics of the blue-breasted fairy-wren, Malurus pulcherrimus, in fragmented habitat in the Western Australian wheatbelt. - Wildl. Res. 29: 225-233.

Brouwer, L. et al. 2011. Strategic promiscuity helps avoid inbreeding at multiple levels in a cooperative breeder where both sexes are philopatric. - Mol. Ecol. 20: 4796-4807.

Cai, W. et al. 2011. Teleconnection pathways of ENSO and the IOD and the mechanisms for impacts on Australian rainfall. - J. Clim. 24: 3910-3923.

Chen, I.-C. et al. 2011. Rapid range shifts of species associated with high levels of climate warming. - Science 333: 1024-1026.

Climate Prediction Center 2012. Monitoring and data: current monthly atmospheric and sea surface temperatures index values. $-<$ www.cpc.ncep.noaa.gov/data/indices $/>$, accessed 1 January 2012.

Cockburn, A. et al. 2008. Can we measure the benefits of help in cooperatively breeding birds: the case of superb fairy-wrens Malurus cyaneus? - J. Anim. Ecol. 77: 430-438. 
Colombelli-Négrel, D. and Kleindorfer, S. 2008. Nest height, nest concealment, and predator type predict nest predation in superb fairy-wrens (Malurus cyaneus). - Ecol. Res. 24: 921-928.

Forchhammer, M. C. et al. 1998. Breeding phenology and climate. - Nature 391: 29-30.

Gardner, J. L. et al. 2011. Declining body size: a third universal response to warming? - Trends Ecol. Evol. 26: 285-291.

Grosbois, V. et al. 2006. Climate impacts on Mediterranean blue tit survival: an investigation across seasons and spatial scales. - Global Change Biol. 12: 2235-2249.

Hallett, T. B. et al. 2004. Why large-scale climate indices seem to predict ecological processes better than local weather. - Nature 430: 71-75.

Hurell, J. W. and Trenberth, K. E. 2010. Climate change. Effects of climate change on birds. - Oxford Univ. Press.

Jenouvrier, S. et al. 2009. Demographic models and IPCC climate projections predict the decline of an emperor penguin population. - Proc. Natl Acad. Sci. USA 106: 1844-1847.

Keith, D. A. et al. 2008. Predicting extinction risks under climate change: coupling stochastic population models with dynamic bioclimatic habitat models. - Biol. Lett. 4: 560-563.

Kingma, S. et al. 2009. Radical loss of an extreme extra-pair mating system. - BMC Ecol. 9: 15.

Lack, D. 1947. The significance of clutch-size. - Ibis 89: 302-352.

Langmore, N. E. et al. 2009. Flexible cuckoo chick-rejection rules in the superb fairy-wren. - Behav. Ecol. 20: 978-984.

Lima, M. et al. 2008. Non-linear feedback processes and a latitudinal gradient in the climatic effects determine green spruce aphid outbreaks in the UK. - Oikos 117: 951-959.

Macmynowski, D. P. et al. 2007. Changes in spring arrival of nearctic-neotropical migrants attributed to multiscalar climate. - Global Change Biol. 13: 2239-2251.

Martinez-Jauregui, M. et al. 2009. Are local weather, NDVI and NAO consistent determinants of red deer weight across three contrasting European countries? - Global Change Biol. 15: 1727-1738.

Mysterud, A. et al. 2000. Relationships between sex ratio, climate and density in red deer: the importance of spatial scale. - J. Anim. Ecol. 69: 959-974.

Nias, R. and Ford, H. 1992. The influence of group size and habitat on reproductive success in the superb fairy-wren Malurus cyaneus. - Emu 92: 238-243.

OOPC 2012. State of the Ocean climate. $-<$ http://stateof theocean.osmc.noaa.gov/sur/ind/dmi.php $>$, accessed 1 January 2012.

Parmesan, C. and Yohe, G. 2003 A globally coherent fingerprint of climate change impacts across natural systems. - Nature 421: 37-42.

Phillimore, A. B. et al. 2010. Differences in spawning date between populations of common frog reveal local adaptation. - Proc. Natl Acad. Sci. USA 107: 8292-8297.

Post, E. and Stenseth, N. C. 1999. Climatic variability, plant phenology, and northern ungulates. - Ecology 80: 1322-1339.

Post, E. et al. 1997. Global climate change and phenotypic variation among red deer cohorts. - Proc. R. Soc. B 264: 1317-1324.

Pruett-Jones, S. et al. 2010. The effects of sex, age, and social status on annual survival in the splendid fairy-wren. - Condor 112: 369-377.

Rainio, K. et al. 2006. Climatic responses in spring migration of boreal and arctic birds in relation to wintering area and taxonomy. - J. Avian Biol. 37: 507-515.

Risbey, J. S. et al. 2009. On the remote drivers of rainfall variability in Australia. - Mon. Weath. Rev. 137: 3233-3253.
Rowley, I. C. R. 1964. The life history of the superb blue wren, Malurus cyaneus. - Emu 64: 251-297.

Rowley, I. C. R. and Russell, E. 1993. The purple-crowned fairywren Malurus coronatus 2: breeding biology, social-organization, demography and management. - Emu 93: 235-250.

Rowley, I. C. R. and Russell, E. 1995. The breeding biology of the white-winged fairy-wren Malurus leucopterus leuconotus in a Western Australian coastal heathland. - Emu 95: 175-184.

Rowley, I. C. R. and Russell, E. M. 2002. A population study of the blue-breasted fairy-wren Malurus pulcherrimus at Dryandra, Western Australia. - Emu 102: 127-135.

Rowley, I. C. R. et al. 1991. The breeding biology of the splendid fairy-wren Malurus splendens: the significance of multiple broods. - Emu 91: 197-221.

Russell, E. and Rowley, I. C. R. 2000. Demography and social organisation of the red-winged fairy-wren, Malurus elegans. - Aust. J. Zool. 48: 161-200.

Sæther, B.-E. et al. 2003. Climate variation and regional gradients in population dynamics of two hole-nesting passerines. - Proc. R. Soc. B 270: 2397-2404.

Saji, N. H. et al. 1999. A dipole mode in the tropical Indian Ocean. - Nature 401: 360-363.

Sandvik, H. and Erikstad, K. 2008 Seabird life histories and climatic fluctuations: a phylogenetic-comparative time series analysis of North Atlantic seabirds. - Ecography 31: 73-83.

Sandvik, H. et al. 2008. A latitudinal gradient in climate effects on seabird demography: results from interspecific analyses. - Global Change Biol. 14: 703-713.

Sandvik, H. et al. 2012. Inter-specific evidence that population dynamics in seabirds are affected by climate both via offspring reproduction and adult survival. - Mar. Ecol. Prog. Ser. 454: 273-284.

Sanz, J. J. 2002. Climate change and breeding parameters of great and blue tits throughout the western Palaearctic. - Global Change Biol. 8: 409-422.

Sanz, J. J. 2003. Large-scale effect of climate change on breeding parameters of pied flycatchers in western Europe. - Ecography 26: $45-50$.

Stenseth, N. C. and Mysterud, A. 2005. Weather packages: finding the right scale and composition of climate in ecology. - J. Anim. Ecol. 74: 1195-1198.

Thomas, C. D. et al. 2004. Extinction risk from climate change. - Nature 427: 145-148.

van Doorn, A. 2007. Ecology and conservation of the purplecrowned fairy-wren (Malurus coronatus coronatus) in the Northern Territory, Australia. - PhD thesis, Univ. of Florida.

van de Pol, M. and Wright, J. 2009. A simple method for distinguishing within- versus between-subject effects using mixed models. - Anim. Behav. 77: 753-758.

van de Pol, M. and Cockburn, A. 2011. Identifying the critical climatic time window that affects trait expression. - Am. Nat. 177: 698-707.

van de Pol, M. et al. 2010. Effects of climate change and variability on population dynamics in a long-lived shorebird. - Ecology 91: 1192-1204.

van de Pol, M. et al. 2011. Poor environmental tracking can make extinction risk insensitive to the colour of environmental noise. - Proc. R. Soc. B 278: 3713-3722.

van de Pol, M. et al. 2012. Fluctuations in population composition dampen the impact of phenotypic plasticity on trait dynamics in superb fairy-wrens. - J. Anim. Ecol. 81: 411-422.

Webster, M. S. et al. 2010. Dealing with uncertainty: flexible reproductive strategies by a tropical passerine bird in an unstable environment. - Adv. Stud. Behav. 42: 123-153. 